Revista Brasileira de Meteorologia, v.27, n.1, 39 - 48, 2012

\title{
COMPORTAMENTO DO VENTO NO SETOR LESTE DE SANTA CATARINA SOB INFLUENCIA DE CICLONES EXTRATROPICAIS
}

\author{
CAMILA DE SOUZA CARDOSO ${ }^{1}$, DANIEL PIRES BITENCOURT ${ }^{1}$ E MAGALY MENDONÇA $^{2}$ \\ ${ }^{1}$ Fundação Jorge Duprat Figueiredo de Segurança e Medicina do Trabalho, Centro Estadual de Santa \\ Catarina (FUNDACENTRO/CESC), Florianópolis, SC, Brasil, \\ ${ }^{2}$ Universidade Federal de Santa Catarina (UFSC), Departamento de Geociências, Florianópolis, SC, Brasil \\ k_mila_krdoso@hotmail.com,daniel@fundacentro.sc.gov.br, magaly@cfh.ufsc.br
}

Recebido Março de 2011 - Aceito Agosto de 2011

\begin{abstract}
RESUMO
A faixa leste catarinense é caracterizada pela presença de terrenos elevados, representados por planaltos e seqüência de serras costeiras subparalelas, cujas altitudes superiores a 1000m decrescem em direção ao oceano. Esta faixa está frequentemente submetida à atuação dos ciclones extratropicais, que geralmente ocasionam ventos fortes causando prejuízos as cidades litorâneas e proximidades. Neste trabalho, o vento observado no leste de Santa Catarina foi analisado para os casos com presença de ciclones extratropicais próximos da costa. O objetivo foi identificar como os ciclones e os fatores locais, como o relevo, têm contribuído para a ocorrência de vento. Selecionaram-se os ciclones extratropicais que atuaram nas proximidades da costa, entre 1998 e 2008. A influência desses sistemas no comportamento do vento foi avaliada através de cálculos de correlação entre a profundidade do ciclone e velocidade do vento observado em sete estações meteorológicas. A contribuição das características locais foi analisada separadamente em cada estação meteorológica, através da análise da freqüência de direção do vento. Os resultados mostraram que estatisticamente, somente os ciclones mais intensos influenciam no comportamento dos ventos. Nos casos de ciclones menos intensos foi verificado que as características do relevo, os efeitos de brisas e a posição do ciclone, em relação à estação, apresentam aproximadamente a mesma importância no comportamento do vento.
\end{abstract}

Palavras-chave: Leste catarinense, comportamento do vento, ciclone extratropical.

\begin{abstract}
BEHAVIOR OF THE WIND IN THE EASTERN SANTA CATARINA UNDER INFLUENCE OF EXTRATROPICAL CYCLONES

The eastern Santa Catarina state is characterized by high level terrain, with plateau and subparallel coastal mountains, whose altitude above $1000 \mathrm{~m}$ decreases toward the ocean. The eastern Santa Catarina is often affected by extratropical cyclones. Generally, this system produces high wind speeds and causes socio-economic losses at coastal cities. In this study, the observed wind in the eastern Santa Catarina was analyzed for cases with presence of extratropical cyclones near to the coast. The purpose was to identify how the cyclones and local factors, as topography, have contributed to the occurrence of wind. Cyclones over an area near to the coast were selected for the period from 1998 to 2008. The influence of the cyclones on the wind was analyzed through the correlations between cyclone depth and wind speed observed at seven meteorological stations. The contribution from local factors was studied through frequency analysis of wind direction for each meteorological station. The results showed that statistically, only strong cyclones have influence over the wind. For cases of weak cyclones, it was verified that the characteristics of topography, effects of breeze, and cyclone offshore distance have shown almost the same influence on wind.
\end{abstract}

Keywords: Eastern Santa Catarina, behavior of wind, extratropical cyclone. 


\section{INTRODUÇÃO}

O estado de Santa Catarina apresenta de leste para oeste três grandes unidades topográficas: as Planícies Costeiras, as Serras Litorâneas e o Planalto Ocidental. Tem $77 \%$ de seu território acima de $300 \mathrm{~m}$ de altitude, com os planaltos entre $300 \mathrm{~m}$ e $900 \mathrm{~m}$, totalizando $56,22 \%$ e as serras situadas acima de $900 \mathrm{~m}$, abrangendo $20,45 \%$ da área total (Nimer, 1979). As Planícies Costeiras estão em contato direto com o oceano Atlântico, as Serras ficam dispostas obliquamente à linha de costa e o Planalto constitui a unidade mais continental e extensa do território catarinense (Rosa e Herrmann, 1986).

A região costeira tem uma extensão de $461,4 \mathrm{~km}$ e é frequentemente atingida por ciclones extratropicais que causam impactos, como vento e agitação marítima, por vezes, bem abrangentes. Os ciclones extratropicais desenvolvem-se em latitudes médias, deslocando-se para sudeste nos três ciclos de vida, em uma distância de aproximadamente $2700 \mathrm{~km}$, e o tempo médio de vida de 3 dias (Sinclair, 1994; Sinclair, 1995; Reboita, 2008; Bitencourt, 2010). A costa leste da Argentina, Uruguai e regiões Sul e Sudeste do Brasil são favoráveis à ocorrência de ciclogêneses (Taljaard, 1967; Ferreira, 1988; Gan e Rao, 1991; Sinclair, 1994; Sinclair, 1995; Hoskins e Hodges, 2005; Mendes, 2006), que atuam durante o ano inteiro, sendo mais freqüentes durante o inverno (Gan e Rao, 1991; Sinclair, 1994; Sinclair, 1995; Mendes et. al., 2009). Murray e Simmonds (1991b) verificaram um máximo de atividade ciclônica durante o verão, na costa leste da América do Sul, entre as latitudes de $20^{\circ} \mathrm{S}$ e $30^{\circ} \mathrm{S}$.

Alguns artigos científicos estudam a estrutura do vento em sistemas vale/montanha (Whiteman, 1982; Whiteman et al., 1999; Whiteman, 2000). Estas circulações ocorrem especialmente em áreas de relevo acidentado, com grandes desníveis como é o caso do setor leste catarinense. A interação de forçantes de escala sinótica com os efeitos locais, produzido pelas elevações resultantes de relevos dissecados, tem sido estudada a partir da década de 80 (Banta e Cotton, 1981; Banta, 1984). No Brasil, estudos que abordam a circulação de ventos em regiões de terreno complexo foram desenvolvidos em Nova Roma do Sul, na região do Vale do Rio da Prata, estado do Rio Grande do Sul, nos trabalhos de Acevedo et al. (2005), Bitencourt e Acevedo (2008). Bitencourt et al. (2009) estudaram a interação dessas circulações com forçantes oriundas de sistemas de grande escala. Whiteman e Doran (1993) mostraram que a existência de circulação forçada termicamente sobre qualquer vale depende da magnitude dos ventos sinóticos de grande escala.

O setor leste da Região Sul do Brasil é fortemente influenciado pela atuação de ciclones extratropicais, e os impactos sócio-econômicos gerados estão associados à ocorrência de precipitação, ventos fortes e agitação marítima. Para essa região há poucos estudos que avaliam especificamente os impactos nos campos de ventos, quando há ocorrência de ciclones extratropicais. Um estudo recente, apresentado por Bitencourt et al. (2010), identificou que a velocidade do vento é estatisticamente influenciada por ciclones extratropicais somente ao sul de $28^{\circ} \mathrm{S}$ de latitude. De acordo com Feitosa et al. (2001), a costa leste da região Sul do Brasil apresenta ventos mais intensos em relação às áreas mais continentais, e isso muito provavelmente esteja relacionado com ciclones extratropicais, principalmente os que atuam sobre o Oceano Atlântico.

O objetivo deste trabalho é avaliar o comportamento do vento observado no setor leste de Santa Catarina, quando há atuação de ciclones extratropicais, nas proximidades do litoral catarinense. Busca-se identificar qual a contribuição dos fatores locais, como o relevo e a maritimidade, que somados às forçantes provenientes dos ciclones, muitas vezes causam ventos fortes com danos e prejuízos a diversos setores.

\section{DADOS E METODOLOGIA}

\subsection{Dados}

Para identificação dos ciclones extratropicais foram utilizados dados de pressão ao nível médio do mar $(p)$, em hPa, obtidos da reanálise do NCEP/NCAR (National Centers for Environmental Prediction/National Centers for Atmospheric Research). Esta reanálise é construída a partir de dados meteorológicos de superfície em terra, navio, radiossonda, aeronaves, satélite e outros, controlando a qualidade e assimilação de dados desde 1957 (Kalnay et al., 1996). A reanálise, acessada em http://www.cdc.noaa.gov/cdc/data.ncep. reanalysis.html, é apresentada numa grade global com resolução espacial de $2,5^{\circ}$ latitude $\times 2,5^{\circ}$ longitude nos horários das 0000 , 0600, 1200 e 1800 UTC.

Para analisar o comportamento do vento foram utilizados dados meteorológicos em três horários diários 0000, 1200, 1800 UTC de velocidade $\left(V_{o b s}\right)$ e direção do vento observado nas estações meteorológicas convencionais de Joinville, Indaial, Itajaí, São José, Lages, São Joaquim e Urussanga, todas distribuídas ao longo do setor leste do estado de SC (Figura 1). Os dados usados foram fornecidos pela Empresa de Pesquisa Agropecuária e Extensão Rural de Santa Catarina S. A. (EPAGRI). A latitude, longitude e altitude dessas estações são apresentadas na Tabela 1. Ressalta-se que o horário das 0600 UTC não foi utilizado por não ocorrer observação meteorológica nas estações administradas pela EPAGRI.

O período da série histórica utilizada inicia em 01 de janeiro de 1998 e finaliza em 20 de outubro de 2008. As falhas contidas nos dados não foram preenchidas, com isso todas as 


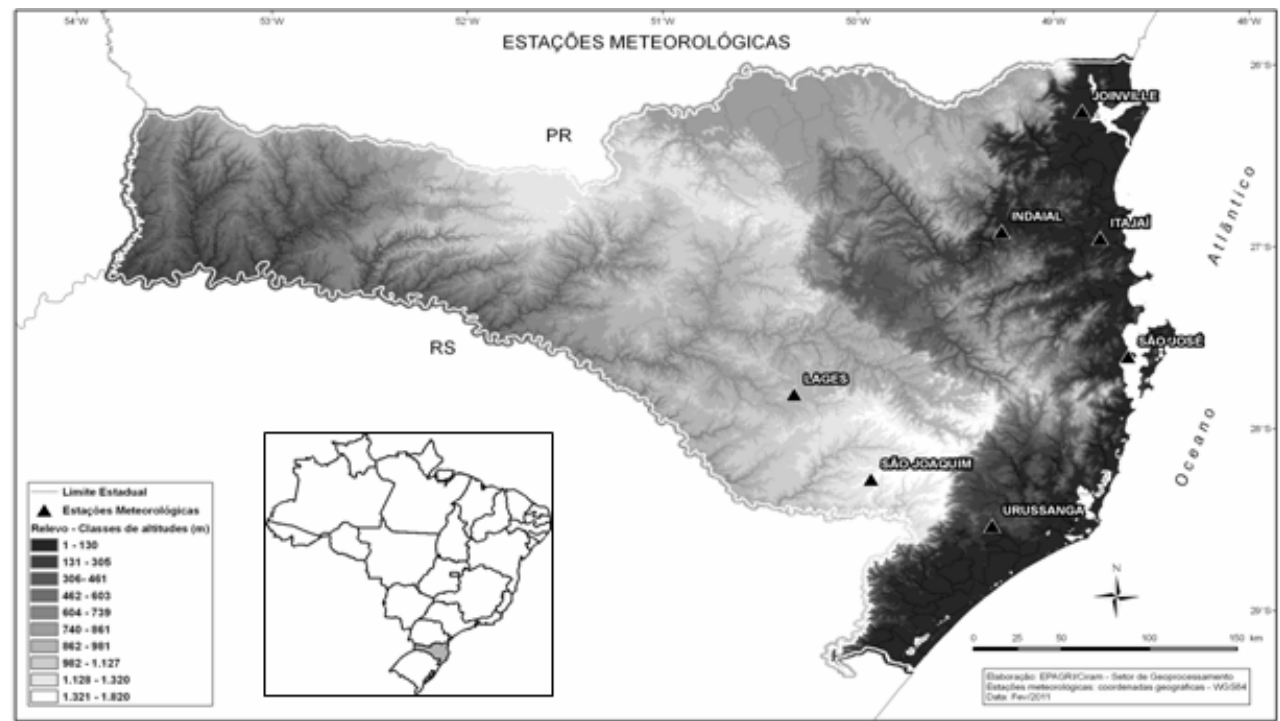

Figura 1 - Mapa do relevo do estado de Santa Catarina com a localização das estações meteorológicas.

Tabela 1 - Estações Meteorológicas Convencionais.

\begin{tabular}{lllll}
\hline Mesorregião & Estação & Latitude & Longitude & Altitude \\
\hline Norte Catarinense & Joinville & $26^{\circ} 15^{\prime} 08^{\prime \prime}$ & $48^{\circ} 51^{\prime} 22^{\prime \prime}$ & $20 \mathrm{~m}$ \\
\hline Vale do Itajaí & Indaial & $26^{\circ} 54^{\prime} 48^{\prime \prime}$ & $49^{\circ} 16^{\prime} 02^{\prime \prime}$ & $86,13 \mathrm{~m}$ \\
\hline Vale do Itajaí & Itajaí & $26^{\circ} 57^{\prime} 01^{\prime \prime}$ & $48^{\circ} 45^{\prime} 41^{\prime \prime}$ & $5 \mathrm{~m}$ \\
\hline Grande Florianópolis & São José & $27^{\circ} 36^{\prime} 07^{\prime \prime}$ & $48^{\circ} 37^{\prime} 11^{\prime \prime}$ & $2 \mathrm{~m}$ \\
\hline Serrana & Lages & $27^{\circ} 48^{\prime} 27^{\prime \prime}$ & $50^{\circ} 19^{\prime} 44^{\prime \prime}$ & $937,73 \mathrm{~m}$ \\
\hline Serrana & São Joaquim & $28^{\circ} 16^{\prime} 31^{\prime \prime}$ & $49^{\circ} 56^{\prime} 03^{\prime \prime}$ & $1376 \mathrm{~m}$ \\
\hline Sul Catarinense & Urussanga & $28^{\circ} 31^{\prime} 55^{\prime \prime}$ & $49^{\circ} 18^{\prime} 53^{\prime \prime}$ & $48 \mathrm{~m}$ \\
\hline
\end{tabular}

avaliações são realizadas apenas nos casos em que há registro de vento.

\subsection{Metodologia}

Os ciclones foram identificados através do uso do esquema automático de identificação de ciclones proposto por Murray e Simmonds (1991a, b). A vantagem de se utilizar um esquema automático de identificação se deve a facilidade em trabalhar com uma grande quantidade de dados, eliminando possíveis erros cometidos em análises subjetivas de cartas sinóticas e imagens de satélite (Murray e Simmonds, 1991a, b; Simmonds e Murray, 1999). O esquema foi desenvolvido na universidade de Melbourne e consiste basicamente em utilizar dados, em pontos de grade, de pressão ao nível médio do mar $(p)$, como os dados de reanálise utilizados neste estudo, para localizar os centros de baixa pressão. Suas trajetórias são rastreadas do início até o final (da ciclogênese até a ciclólise), além de várias outras estatísticas de sua distribuição e movimento, como densidade espacial, velocidade, pressão central e tendência de pressão dos ciclones, e freqüência de ciclogêneses e ciclólises (Murray e Simmonds, 1991 a, b). O algoritmo foi aperfeiçoado no final da década de 90 e todos os detalhes dessas melhorias são mostrados por Simmonds e Murray (1999) e Simmonds et al. (1999).

O algoritmo de identificação e rastreamento de ciclones, proposto pela Universidade de Melbourne, possibilita que sejam localizados sistemas fechados ou abertos. Os ciclones fechados são identificados pela minimização da pressão, enquanto que os sistemas abertos são identificados através de interpolação de pontos de inflexão na superfície de pressão interpolada, e localizados minimizando-se o valor absoluto do gradiente de pressão (Murray e Simmonds, 1991b). Para este trabalho, o algoritmo cedido pela Universidade de Melbourne foi definido para identificar somente os sistemas ciclônicos fechados, que atuaram na região compreendida entre as latitudes $25^{\circ} \mathrm{S}$ e $31^{\circ} \mathrm{S}$ e longitudes $42^{\circ} \mathrm{W}$ e $52^{\circ} \mathrm{W}$, sendo descartados os sistemas com o laplaciano da pressão $\left(\nabla^{2} p\right)$ menor que $0,2 \mathrm{hPa}$ lat $^{-2}$. Utilizou-se nas análises a profundidade $(D)$ do sistema, dada pela Equação 1:

$$
D=\frac{1}{2} \frac{\partial^{2} p}{\partial r^{2}} R^{2}=\frac{1}{4} \nabla^{2} p R^{2}
$$


onde $\nabla^{2} p$ é o valor médio do laplaciano da pressão, numa área correspondente a região que cerca o máximo $\nabla^{2} p$, em que o $\nabla^{2} p$ é positivo. A distância média, em ${ }^{\circ}$ lat, entre o centro do sistema e a periferia dessa área é o raio $(R)$ do sistema. A profundidade $(D)$ é representativa da influência e intensidade do ciclone numa determinada escala espacial, pois relaciona $\nabla^{2} p$ e o $R$ do sistema (Simmonds e Keay, 2000a; Lim e Simmonds, 2007). Um ciclone, com uma determinada profundidade, apresentará maior intensidade $\left(\nabla^{2} p\right)$, quanto menor for a escala horizontal (Simmonds e Keay, 2000a).

O efeito dos sistemas ciclônicos para cada estação meteorológica é avaliado através do cálculo da correlação de Pearson (r), entre a profundidade $(D)$ e o $V_{o b s}$. O cálculo de $\mathrm{r}$ primeiramente foi elaborado para toda a série de sistemas ciclônicos identificados na área de estudo. Posteriormente, calculou-se r separadamente para cada estação do ano (inverno, primavera, verão e outono) e também somente para os ciclones mais intensos (quarto quartil). Classificou-se como estação de verão os meses de dezembro, janeiro e fevereiro (DJF); outono, os meses de março, abril e maio (MAM); inverno, os meses de junho, julho e agosto (JJA); e primavera os meses de setembro, outubro e novembro (SON). O coeficiente de correlação de Pearson (r) é dado pela Equação 2:

$$
\mathrm{r}=\frac{\sum_{i=1}^{n} X_{i} Y_{i}}{\left[\left(\sum_{i=1}^{n} X_{i}^{2}\right)\left(\sum_{i=1}^{n} Y_{i}^{2}\right)\right]^{1 / 2}}
$$

onde $n$ é o número de horários em que foi identificado ciclone extratropical na área mostrada da figura 2, $X$ representa os valores de profundidade $D$, e $Y$ representa o $V_{o b s}$. Neste trabalho será considerada a classificação das correlações de Pearson como fraca $(0 \leq \mathrm{r}<0,3)$, moderada $(0,3 \leq \mathrm{r}<0,7)$ e forte $(0,7 \leq \mathrm{r}<1)$ (Maxwell e Satake, 1997). Utilizando o nível de significância de $5 \%(\mathrm{P} \leq 0,05)$, aplicou-se nos resultados de r o teste $t$ de Student (Spiegel et al., 2000).

A avaliação da velocidade e direção do vento foi realizada somente para os casos que apresentaram significância estatística. A associação do $V_{o b s}$ e $D$ foi apresentada para a série de $D$ dividida em percentis de $25 \%$, em que se considerou o primeiro e quarto quartis da $D$, como ciclones menos intensos e mais intensos, respectivamente. A avaliação da direção do vento predominante foi elaborada através do cálculo de freqüência da direção do vento observado.

\section{RESULTADOS E DISCUSSÕES}

\subsection{Avaliação da velocidade do vento observado}

Considerando todas as situações com presença de ciclone extratropical próximo da costa catarinense, verifica-se que as associações de $D$ com o $V_{o b s}$ na série histórica são fracas (Tabela 2). Em geral nas estações localizadas em latitudes mais ao sul, os valores de r aumentam, sendo São Joaquim a localidade que apresenta as melhores associações, possivelmente pelo fato de ser a estação mais elevada, numa altitude de $1346 \mathrm{~m}$, e sem barreiras impostas pelo relevo, que impedem a circulação de ventos. Urussanga, embora seja a estação que está posicionada mais ao sul e mais próxima do litoral, apresenta $\mathrm{r} \approx 0,12$, ou seja, fraca associação entre $D$ e $V_{o b s}$. A exceção é São José que apresentou baixo valor de correlação e sem significância estatística. Bitencourt et al. (2010), embora tenham utilizado uma área mais abrangente para identificar os ciclones e dados de estações meteorológicas automáticas com observações a cada hora, encontraram associações semelhantes para o caso de Urussanga e São José e atribuíram ao relevo, a leste das estações, como principal causa.

A análise sazonal (Tabela 2) mostra que São Joaquim é a única estação que apresenta associação entre a $D$ do ciclone e o $V_{o b s}$ nas quatro estações do ano, sendo que existe correlação fraca no verão, outono e primavera, e moderada no inverno, com $r$ de 0,37. A melhor associação da $D$ com o $V_{o b s}$ durante o inverno é explicada pelo fato dos ciclones serem mais intensos (Simmonds e Keay, 2000b), já que a baroclinia é maior nesta época. Para a estação de Itajaí, há correlação moderada entre $D$ e $V_{o b s}$ para o outono, inverno e primavera, com valores de r pouco maiores no outono. Neste caso não há correlação somente no verão, o que sugere que os efeitos de brisa, forçada pela diferenciação térmica terra-mar nesta época do ano, prevaleçam e minimizem os efeitos dos sistemas ciclônicos, que em geral, são mais fracos.

Os baixos valores de correlação e a ausência de associação em determinadas estações do ano em algumas

Tabela 2 - Correlação (r) entre a profundidade (D) do ciclone e o vento observado (Vobs) para cada estação meteorológica.

\begin{tabular}{lccccccc}
\hline \multirow{2}{*}{$(\mathbf{P}<\mathbf{0 , 0 5})$} & \multicolumn{7}{c}{ Estações Meteorológicas } \\
\cline { 2 - 8 } & Joinville & Indaial & Itajaí & $\begin{array}{c}\text { São } \\
\text { José }\end{array}$ & Lages & São Joaquim & Urussanga \\
\hline Série histórica & 0,12 & 0,21 & 0,24 & 0,07 & 0,2 & 0,29 & 0,12 \\
Verão (DJF) & 0,03 & $-0,00$ & $-0,02$ & 0,22 & $-0,03$ & 0,20 & 0,06 \\
Outono (MAM) & 0,29 & 0,20 & 0,39 & 0,06 & 0,21 & 0,26 & 0,10 \\
Inverno (JJA) & 0,26 & 0,30 & 0,32 & 0,16 & 0,44 & 0,37 & 0,10 \\
Primavera (SON) & 0,03 & 0,35 & 0,34 & $-0,08$ & 0,21 & 0,29 & 0,26 \\
\hline
\end{tabular}


localidades, como as estações de Joinville, São José e Urussanga (Tabela 2), indicam que a presença de forçantes locais, como o relevo, cobertura do solo, desenho do litoral de $\mathrm{SC}$ e a diferenciação térmica favorecida pelos contrastes terra/mar e vale/montanha, influenciam o comportamento do vento observado. Em outras palavras, mesmo com a presença de ciclones extratropicais próximos à costa catarinense (Figura 2a), as características locais em torno de cada estação

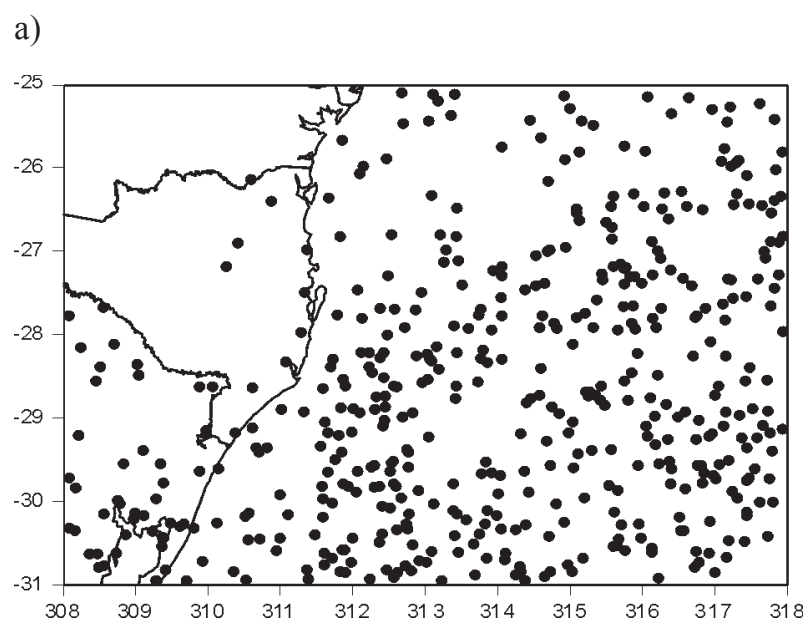

b)

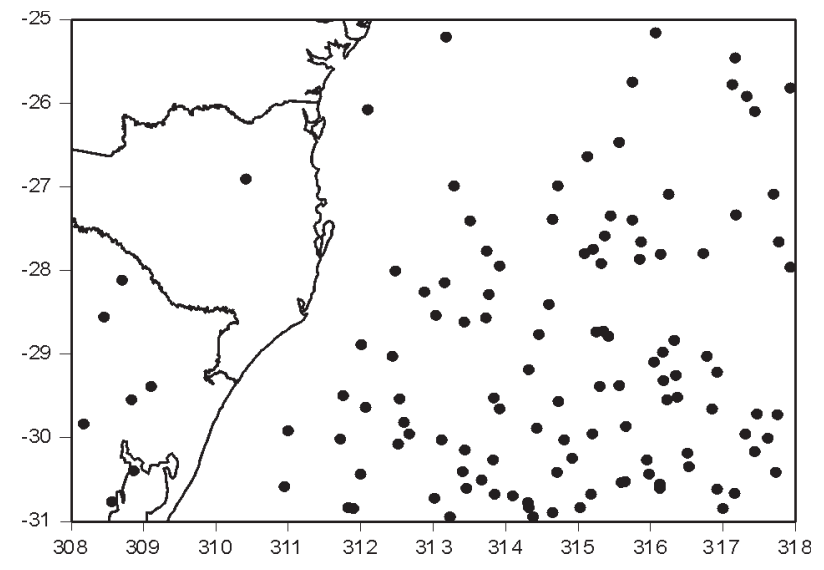

Figura 2 - Densidade (a) de todos os ciclones e (b) dos mais intensos $\left(4^{\circ}\right.$ quartil de D) identificada entre 01 de janeiro de 1998 a 20 de outubro de 2008 . meteorológica mostram-se bastante importantes para o comportamento dos ventos.

Separando os casos de ciclones mais intensos ( $4^{\circ}$ quartil) que ocorreram próximo do litoral de SC verifica-se que a estação meteorológica de São José não apresenta correlação com significância estatística na série histórica (Tabela 3). Esse resultado é concordante com Bitencourt et al. (2010), que identificaram ausência de associação entre os ciclones e velocidade do vento nessa faixa latitudinal. Em geral, os ciclones mais intensos ocorrem em áreas mais afastadas da costa (Figura 2b), proporcionando um aumento da importância das características locais em torno da estação, como forçantes para a ocorrência de ventos. Os ciclones se intensificam à medida que se deslocam para sudeste (em direção ao oceano), onde os gradientes de temperatura da superfície do mar geralmente são maiores (Sinclair, 1995).

A importância dos ciclones mais intensos, como causa do vento observado, pode ser verificado através da Figura 3 , a qual mostra que a média e a mediana do $V_{o b s}$ são sempre maiores para o quarto quartil em todas as estações meteorológicas. Apenas as estações de Joinville e Urussanga apresentam pouca diferença dos valores de $V_{o b s}$, quando se compara os ciclones menos intensos ( $1^{\circ}$ quartil) e mais intensos ( $4^{\circ}$ quartil).

Em São Joaquim, independente da intensidade do ciclone, a média, desvio padrão e o valor máximo do $V_{o b s}$ são mais elevados comparando com as demais estações meteorológicas (Figura 3). Isto reforça que a altitude dessa estação, acima de $1300 \mathrm{~m}$, contribui para ocorrência de maiores valores de $V_{o b s}$, assim como a topografia de planalto que é levemente ondulada. Entretanto, ressalta-se que o vento máximo em quase todas as estações (exceto Itajaí) não ocorre nos casos de ciclones mais intensos (Figura 3). Uma hipótese para isto, é que os ventos fortes sejam causados pelas forçantes locais.

A análise sazonal mostra que a média e a mediana do $V_{o b s}$ também são sempre maiores nos casos de ciclones mais intensos (Figura 4). Durante o verão a análise é feita para São José e São Joaquim, sendo esta a única estação do ano em que a média, mediana, desvio padrão e $V_{o b s}$ máximo são menores para São Joaquim (Figura 4a). Esta particularidade possivelmente está relacionada com o maior gradiente de temperatura terra-mar

Tabela 3 - Correlação (r) entre a profundidade (D) dos ciclones mais intensos (quarto quartil) e o vento observado (Vobs) para cada estação meteorológica.

\begin{tabular}{lccccccc}
\hline \multirow{2}{*}{$(\mathbf{P}<\mathbf{0 , 0 5})$} & \multicolumn{7}{c}{ Estações Meteorológicas } \\
\cline { 2 - 8 } & Joinville & Indaial & Itajaí & São José & Lages & São Joaquim & Urussanga \\
\hline Série histórica & 0,22 & 0,40 & 0,46 & 0,00 & 0,39 & 0,28 & 0,34 \\
Verão (DJF) & $-0,12$ & $-0,05$ & 0,04 & $-0,12$ & $-0,10$ & $-0,08$ & 0,20 \\
Outono (MAM) & 0,21 & 0,49 & 0,42 & $-0,01$ & 0,24 & 0,52 & 0,56 \\
Inverno (JJA) & 0,33 & 0,63 & 0,58 & 0,34 & 0,56 & 0,06 & 0,22 \\
Primavera (SON) & 0,40 & 0,53 & 0,57 & $-0,16$ & 0,51 & 0,36 & 0,33 \\
\hline
\end{tabular}




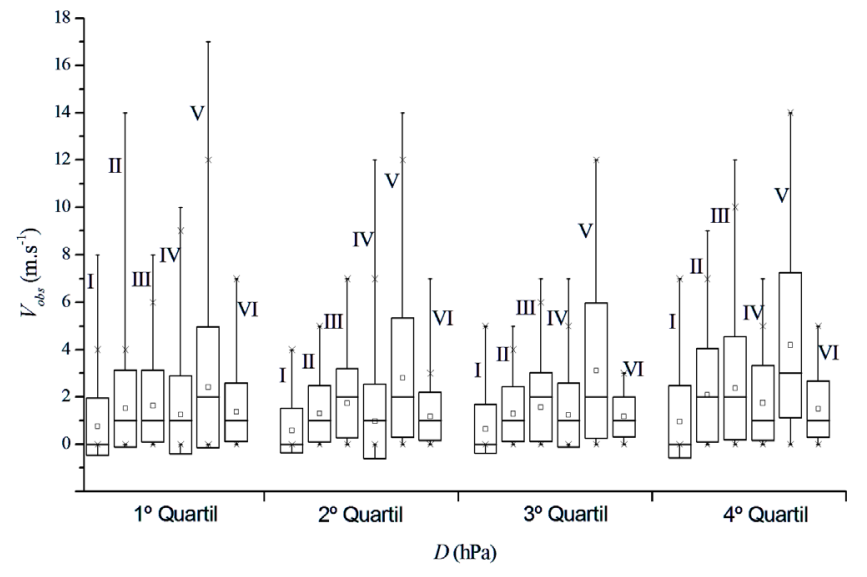

Figura 3: Média ( $\square$ ), mediana ( - no interior da caixa), desvio padrão acima e abaixo da média (caixa), percentis de $1 \%(\times$ inferior $)$ e $99 \%$ $(\times$ superior) e valores mínimo $(\stackrel{\perp}{)}$ e máximo $(T)$ do vento observado (Vobs) para as estações meteorológicas (I) Joinville, (II) Indaial, (III) Itajaí, (IV) Lages, (V) São Joaquim e (VI) Urussanga, como uma função do $1^{\circ}, 2^{\circ}, 3^{\circ}$ e $4^{\circ}$ quartil da profundidade do ciclone $\mathrm{D}(\mathrm{hPa})$.
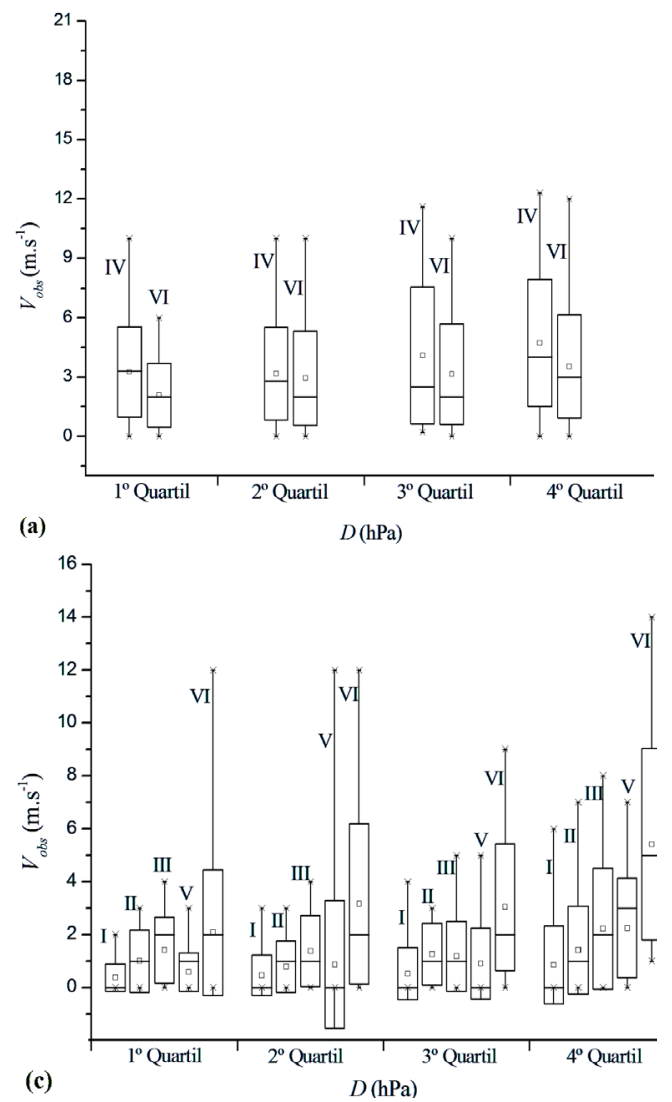

durante o verão, que intensifica a ação das brisas, que podem se somar aos ventos provenientes dos ciclones, resultando em ocorrência de ventos mais intensos nas estações próximas do litoral.

\subsection{Avaliação da direção do vento observado}

A ocorrência de ventos ocasionados por ciclones extratropicais que se deslocam próximos do litoral catarinense são alterados pelas características geográficas do estado. O relevo bastante acidentado com a presença da Serra Geral, escarpa do Planalto Meridional, próxima do litoral faz com que a direção do vento tenha características distintas conforme a localização da estação meteorológica. Monteiro (2007) constatou diferentes padrões de direção de vento para nove estações meteorológicas, instaladas no litoral Sul de SC, encontrando grande variabilidade espacial na direção do vento nessa região.

Semelhanças na direção do vento predominante são verificadas em Indaial, Itajaí e Lages (Figura 5b, 5c e 5d), em que a predominância de ventos de SW sugere uma circulação ao
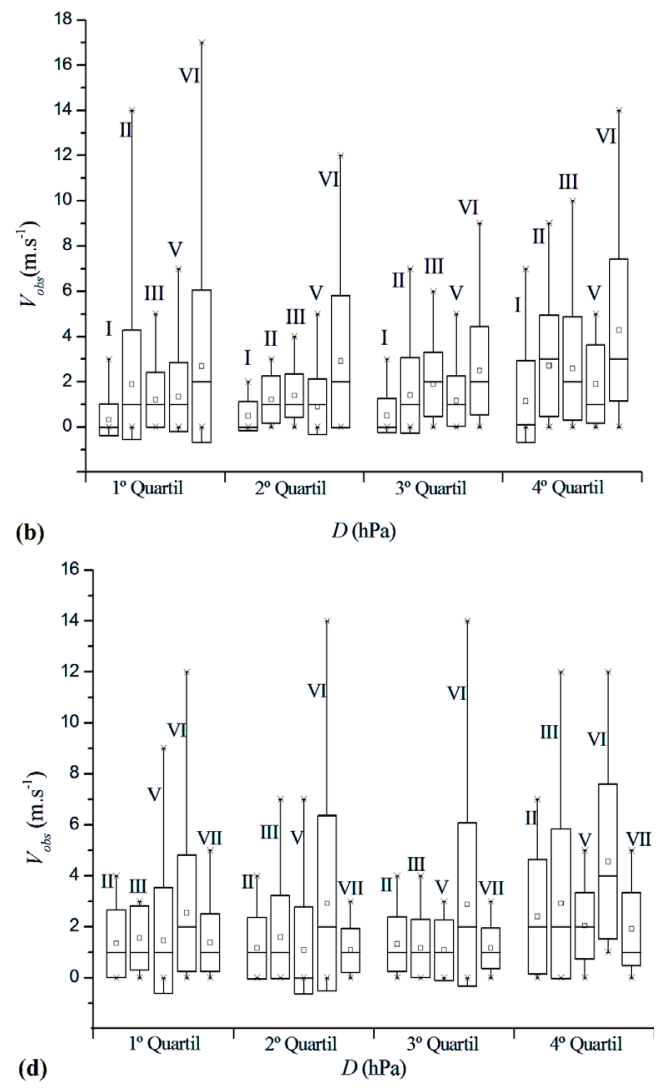

Figura 4 - Média ( $\square)$, mediana ( - no interior da caixa), desvio padrão acima e abaixo da média (caixa), percentis de $1 \%$ ( $\times$ inferior) e 99 \% ( $\times$ superior) e valores mínimo $\left({ }^{\perp}\right)$ e máximo $(T)$ do vento observado (Vobs) para as estações meteorológicas (I) Joinvile, (II) Indaial, (III) Itajaí, (IV) São José, (V) Lages, (VI) São Joaquim e (VII) Urussanga, como uma função do $1^{\circ}, 2^{\circ}, 3^{\circ}$ e $4^{\circ}$ quartil da profundidade do ciclone D (hPa) no (a) verão, (b) outono, (c) inverno e (d) primavera. 

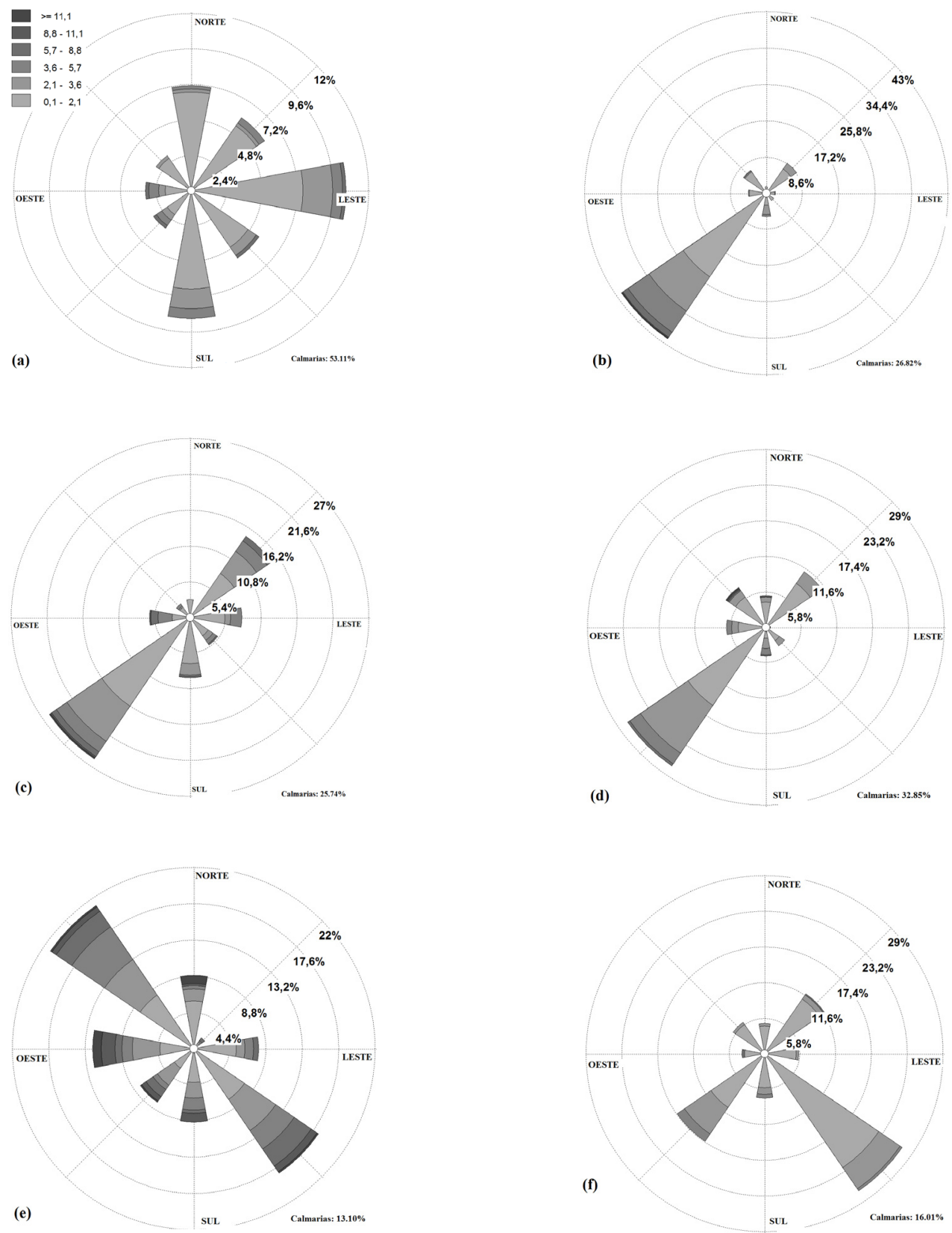

Figura 5 - Direção do vento predominante quando há atuação de ciclones para as estações meteorológicas (a) Joinville, (b) Indaial, (c) Itajaí, (d) Lages, (e) São Joaquim e (f) Urussanga. A escala de velocidade $\left(\mathrm{m}_{\mathrm{s}} \mathrm{s}^{-1}\right)$ é apresentada no primeiro gráfico. Os valores percentuais representam a distribuição de freqüência.

longo da Serra Geral. Esse comportamento também é verificado no caso dos ciclones mais intensos, onde houve diminuição dos ventos de NE e aumento nas ocorrências de $\mathrm{W}$ para as estações de Itajaí e Lages (Figura 6c e 6d).

Joinville está posicionada a sotavento e no sopé da Serra do Mar que pode alcançar os 1500m (Figura 1), estando totalmente protegida dos ventos de W (Figura 5a) que deveriam confluir em direção ao centro dos ciclones. Por ser a estação mais ao norte do litoral catarinense, onde o efeito do vento proveniente de ciclones é menor do que em estações mais ao sul, verifica-se que há pelo menos três predominância de vento observado (Figura 5a), além da maior ocorrência de calmarias.

São Joaquim está mais exposta a ação dos ventos devido sua localização na borda do planalto, na Serra Geral, onde são verificadas as maiores velocidades de vento e a menor ocorrência de calmarias. Os ventos de NW e SE são as duas direções preferências (Figura 5e), em que a presença de escoamentos de NW (21\%) pode estar relacionada à fase de formação e intensificação da baixa em 

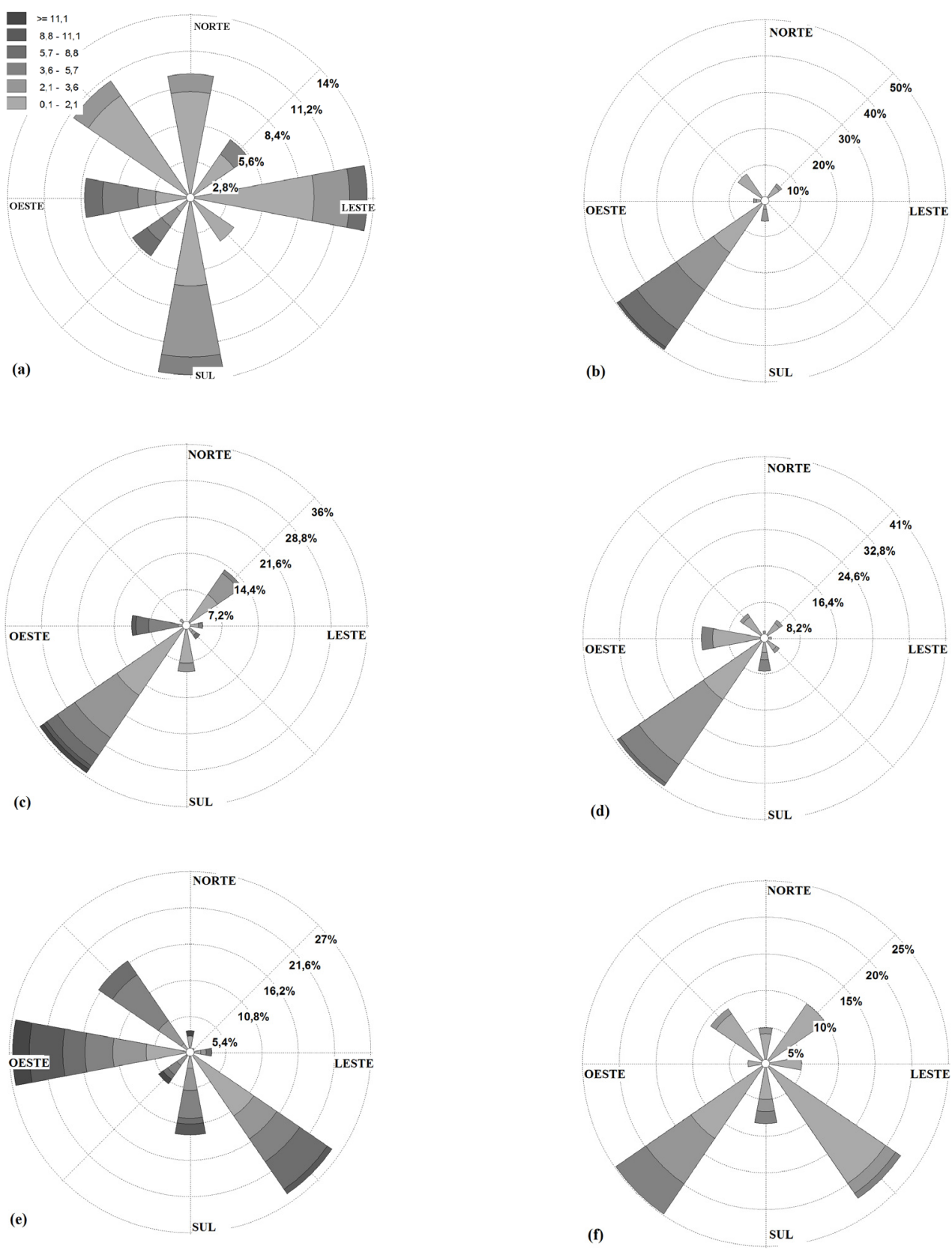

Figura 6 - Direção do vento predominante nos casos de ciclones mais intensos para as estações meteorológicas de (a) Joinville (b) Indaial, (c) Itajaí, (d) Lages, (e) São Joaquim, (f) Urussanga. A escala de velocidade $\left(\mathrm{m}_{\mathrm{s}} \mathrm{s}^{-1}\right)$ é apresentada no primeiro gráfico. Os valores percentuais representam a distribuição de freqüência.

superfície, e também aos ciclones que estão próximos do litoral catarinense. A ocorrência de ventos de SE está associado aos ciclones que ficam mais afastados do litoral. No caso de ciclones mais intensos, percebe-se que houve uma diminuição na ocorrência de ventos de NW, aumentando a freqüência de ventos de W e SE, sendo o W com maior intensidade (Figura 6e).

Urussanga está posicionada no sopé da escarpa da Serra Geral (Figura 1), numa região de relevos com desníveis acentuados, onde a ocorrência de ventos é bastante influenciada pelos efeitos locais. Isto pode ser verificado na figura $5 \mathrm{f}$, onde a direção do vento predominante é de SE, possivelmente proveniente dos ciclones já desenvolvidos e que se deslocam pelo litoral de SC. Sendo assim, as circulações de NW a SW que ocorrem no início do processo da ciclogênese, e que tenderiam a convergir na direção do centro do ciclone, são barrados pela Serra Geral. Para os casos de ciclones mais intensos (Figura 2b) a direção do vento predominante passou a ser de SW, e em seguida de SE (Figura 6f). 
Sazonalmente, a direção do vento não apresentou variações significativas (figura não mostrada). Para o caso de São José, que apresentou associação da $D$ com o $V_{o b s}$ somente no verão, verifica-se que a direção do vento predominante é de $\mathrm{S}$, e em seguida, vento de SE.

\section{RESUMO E CONCLUSÕES}

Foram utilizadas sete estações meteorológicas convencionais, distribuídas no setor leste catarinense para analisar o comportamento do vento observado, quando há atuação de ciclones extratropicais próximo do litoral de SC. Os ciclones foram identificados por meio do esquema automático de identificação e rastreamento proposto por Murray e Simmonds (1991a, b), e as avaliações foram feitas através de cálculos de correlação entre a $D$ e o $V_{o b s}$.

Os resultados apresentados mostraram que embora as associações da $D$ com o $V_{o b s}$ sejam fracas, estatisticamente os ciclones influenciam a ocorrência de ventos no setor leste de SC. As melhores associações foram encontradas em São Joaquim, indicando que a altitude da estação contribui para que a variabilidade na velocidade do vento seja bastante influenciada pelos ciclones. As características do relevo e a diferenciação térmica terra/mar mostraram-se bastante importantes na ocorrência do $V_{o b s}$, especialmente nos casos de ciclones menos intensos. Nos casos de ciclones mais intensos, a associação da $D$ com o $V_{o b s}$ são inexistentes para as estações de Joinville e São José. Em Urussanga o efeito produzido pelo relevo, com a proximidade da Serra Geral a oeste, influencia o comportamento do vento. Esses resultados são concordantes com trabalhos anteriores (Monteiro, 2007; Bitencourt et al., 2010).

A análise da associação entre a $D$ e $V_{o b s}$, quando separada em quartis, mostrou que os ciclones mais intensos ocasionam maiores valores no $V_{\text {obs }}$. Porém, constatou-se também que rajadas fortes de vento podem ocorrer durante a ciclogênese. Sazonalmente, verifica-se que o verão é a estação do ano em que os ciclones exercem menor influencia no $V_{o b s}$.

A avaliação da direção do vento confirmou a importância dos fatores locais e também sugere que se deva considerar a posição do ciclone em relação às estações meteorológicas. A presença da Serra Geral, com altitudes superiores a $1000 \mathrm{~m}$ e obliqua a linha de costa, altera a direção do vento nas estações de Lages, Indaial, Itajaí e Urussanga. Em Joinville nota-se que a presença da Serra do Mar, contribui para inibição da circulação de oeste. A estação de São Joaquim é a que está mais sujeita a ação dos ventos provenientes dos ciclones, devido sua localização na borda da Serra Geral e altitude de 1346m.

Os resultados aqui apresentados mostraram-se bastante importantes para avaliar o efeito dos ciclones extratropicais na ocorrência de ventos no setor leste catarinense, considerando também as características existentes em torno de cada estação meteorológica. A principal conclusão desse trabalho é que o vento observado em cada estação meteorológica é influenciado pelas características locais, mesmo que haja presença de ciclones extratropicais no litoral de SC. Entretanto, sugere-se que trabalhos futuros façam estudos mais detalhados sobre a influência dessas características locais em torno de cada estação meteorológica. Estudos de caso abordando, por exemplo, o efeito da urbanização em torno da estação de São José, com presença de ciclones nas proximidades, poderá refinar os resultados apresentados neste trabalho. Sugere-se ainda a elaboração de pesquisas que abordem a relação entre a intensidade e a posição do ciclone.

\section{AGRADECIMENTOS}

O primeiro autor agradece ao Programa de PósGraduação em Geografia da Universidade Federal de Santa Catarina, sendo este trabalho parte de sua dissertação de mestrado. Os autores agradecem ao Conselho Nacional de Desenvolvimento Científico e Tecnológico - CNPq (Projeto 471917/2008-7) e a Financiadora de Estudos e Projetos FINEP (Projeto 01.09.0295.00) pelo suporte à pesquisa. Ao INMET e a EPAGRI pela cessão dos dados de vento. Ao National Centers for Environmental Prediction (NCEP) pela disponibilização dos dados de reanálise. À Universidade de Melbourne, por conceder o esquema de identificação de ciclones.

\section{REFERÊNCIAS BIBLIOGRÁFICAS}

ACEVEDO, O. C.; et al. EXPANTAS 2005: Experimento de Observações das Transferências entre a Superfície e a Atmosfera na Região do Rio das Antas. Ciência e Natura, Santa Maria, RS, v. esp., p.209-212, 2005.

BANTA, R. M.. Daytime Boundary-Layer Evolution over Mountainous Terrain. Part 1: Observations of the Dry Circulations Monthy Weather Review, Ft. Collins, v. 112, n. 2, p.340-356, 1984.

BANTA, R.; COTTON, W. R.. An Analysis of the Structure of Local Wind Systems in a Broad Mountain Basin. Journal Of Applied Meteorology, v. 20, n. 11, p.1255-1266, 1981. BITENCOURT, D. P.; ACEVEDO, O. C.. Modelling the Interaction Between a River Surface and the Atmosphere at the Bottom of a Valley. Boundary-Layer Meteorology, v. 129, p.309-321, 2008.

BITENCOURT, D.; et al. A interação do vento local no interior de um vale com o escoamento de grande escala - Análise de dois estudo de caso. Revista Brasileira de Meteorologia, v. 24, n. 4 , p. 436 - 447, 2009. 
BITENCOURT, D.; et al.. Relating winds along the Southern Brazilian coast to extratropical cyclones. Meteorological Applications. V. 17 DOI: 10.1002/met.232, 2010.

FEITOSA, E.; PEREIRA, A.; VELEDA, D. Brazilian Wind Atlas Project. In: EUROPEAN WIND ENERGY CONFERENCE, 2001, Copenhage, Dinamarca, Anais ... in CD-ROM, 2001.

FERREIRA, C. C. Ciclogêneses e ciclones extratropicais na região sul-sudeste do Brasil e suas Influencias no tempo. 1988. 118 f. Dissertação(Mestrado em Meteorologia) - Instituto Nacional de Pesquisas Espaciais, São José dos Campos, 1988.

GAN, M.A.; RAO, V. B.: Surface cyclogenesis over SouthAmerica. Monthy Weather Review, v. 119, p.1293-1302. 1991.

$\mathrm{HO} \Sigma \mathrm{KIN} \Sigma$, B. J.; HO $\Delta \Gamma E \Sigma$, K. I.: A new on Southern Hemisphere storm tracks. Journal Climate, v. 18, p. 4108-4129, 2005.

KALNAY, E. et al.. The NCEP/NCAR 40-Year Reanalysis Project. Bulletin of the American Meteorological Society, v. 77, n. 3, p. 437 - 471, 1996.

LIM, E.P.; SIMMONDS, I. Southern Hemisphere Winter Extratropical cyclone Characteristics and Vertical Organization Observed with the ERA-40 Data in 1979-2001. Journal of Climate, v.20, p. 2675-2690, 2007. DOI: 10.1175/JCLI4135.1.

MAXWELL,D.L.; SATAKE,E. Research and statistical methods in communication disorders. USA: William \& Wilkins, 1997.

MENDES, D. Regimes de circulação no Atlântico Sul e sua relação com a localização e intensidade de sistemas activos e com o balanço de vapor na região. 2006. $115 f$. Tese de Doutorado (Doutorado em Física), Universidade de Lisboa, Portugual, 2006.

MENDES, D., SOUZA, E. P., MARENGO, J. A., MENDES, M. C. D.: Climatology of extratropical cyclones over the South American-southern oceans sector. Theor. Appl. Climatol., doi: 10.1007/s00704-009-0161-6, 2009.

MONTEIRO, M. A. Dinâmica Atmosférica e Caracterização dos Tipos de Tempo na Bacia Hidrográfica do Rio Araranguá. 2007. 224F. Tese (Doutorado em Geografia) Programa de Pós-Graduação em Geografia, Universidade Federal de Santa Catarina, Florianópolis, 2007.

MURRAY, R. J.; SIMMONDS, I.: A numerical scheme for tracking cyclone centers from digital data. Part I: Development and operation of the scheme. Australian Meteorological Magazine, v. 39, 155-166, 1991a.

MURRAY, R. J.; SIMMONDS, I. A numerical scheme for tracking cyclone centers from digital data. Part II: Application to January and July general circulation model simulations. Australian Meteorological Magazine, v. 39, 167-180, 1991b.

NIMER. E. Climatologia do Brasil. 2.ed. Rio de Janeiro: Instituto Brasileiro de Geografia e Estatística, 1979. 421p.

REBOITA, M. S.: Ciclones extratropicais sobre o Atlântico Sul: Simulação climática e experimentos de sensibilidade.
2008. 316p. Tese (Doutorado em Meteorologia) Universidade de São Paulo, São Paulo, SP, 2008.

ROSA, R. O.; HERRMANN, M. L. P. Geomorfologia. In: Santa Catarina. GAPLAN. Atlas de Santa Catarina. Rio de Janeiro, Aerofoto Cruzeiro do Sul, 1986.

SIMMONDS, I.; KEAY, K.; Variability of Southern Hemisphere Extratropical Cyclone Behaviour, 1958-97. Journal of Climate, v. 13, 550-561, 2000a.

SIMMONDS, I.; KEAY, K; Mean Southern Hemisphere Extratropical Cyclone Behaviour in the 40-Year NCEPNCAR Reanalysis. Journal of Climate, v. 13, p. 873-885, $2000 b$.

SIMMONDS, I.; MURRAY, R. J.: Southern Extratropical Cyclone Behaviour in ECMWF Analysis during the FROST Special Observing Periods. Weather and Forecasting, v. 14, 878-891, 1999.

SIMMONDS, I.; MURRAY, R. J.; LEIGHTON, R. M.: A refinement of cyclone tracking methods with data from FROST. Australian Meteorological Magazine, Especial Edition, p. 35-49, 1999.

SINCLAIR, M. R.: An Objective cyclone climatology for the Southern Hemisphere. Monthy Weather Review, v. 122, p. 2239-2256, 1994.

SINCLAIR, M. R. A climatology of cyclogenesis for the Southern Hemisphere. Monthy Weather Review, v. 123, p. 1601-1619, 1995

SPIEGEL, M. R.; SCHILLER, J. J.; SRINIVASAN, A. L. Schaum's outline of probability and statistics. New York: McGraw-Hill, 2000.

TALJAARD, J. J. Development, distribution and movement of cyclones and anticyclones in the Southern Hemisphere during IGY. Journal of Applied Meteorology, v.6, p. 973987, 1967

WHITEMAN, C. D.. Breakup of Temperature Inversions in Deep Mountain Valleys: Part I. observations. . Journal of Applied Meteorology, Richland, v. 21, n. 3, p.270-289, 1982.

WHITEMAN, C. D.; DORAN, J. C. The Relationship between Overlying Synoptic-Scale Flows and Winds within a Valley. Journal of Applied Meteorology, Richland, v. 32, n. 11, p.1669-1682, 1993.

WHITEMAN, C. D.; BIAN, X.; SUTHERLAND, J. L.. Wintertime Surface Wind Patterns in the Colorado River Valley. Journal of Applied Meteorology, v. 38, n. 8, p.1118-1130, 1999.

WHITEMAN, C. D.. Mountain Meteorology: Fundamentals and Applications. New York and Oxford: Oxford University Press, 2000. 355 p. 